\title{
El aporte de la Geología en investigaciones arqueológicas multidisciplinarias e interdisciplinarias: casos de estudio
}

\author{
Adriana M. Blasi \\ División Mineralogía y Petrología Museo de La Plata-CIC-UNLP \\ Paseo del Bosque S/N (1900) La Plata, Buenos Aires, Argentina \\ ablasi@fcnym.unlp.edu.ar
}

* Este documento deve ser referido como segue

Blasi A. M. 2008.

El Aporte de la Geología en Investigaciones Arqueológicas Multidisciplinarias e Interdisciplinarias: Casos de Estudio Terræ Didatica, 3(1):36-49.

$<$ http://www.ige.unicamp br/terraedidatica/>

\begin{abstract}
RESUMO Se presentan en esta contribución ejemplos de trabajos de investigación realizados desde la Geología para la resolución de diversos cuestionamientos arqueológicos. Estos se discriminan sobre la base de su carácter en interdisciplinarios y multidisciplinarios. Se valora el grado de compromiso y generación de equipos de investigación en cada caso. Se puntualizan las limitaciones en la generación de este tipo de aportes, en especial la escala temporal y espacial del desarrollo las ocupaciones humanas del territorio Americano y las políticas científicas que desalentaron por mucho tiempo las contribuciones de este sesgo. Se numeran aspectos que son requeridos para contextualizar sitio arqueológico, entre otros, estudios de marcos geográficos, fisiográficos, topográficos, geomorfológicos, geológicos, geocronológicos y pedosedimentarios; así también la necesidad de conocimientos acerca de la naturaleza de las materias primas inorgánicas utilizadas para la realización de la cultura material $y$ sus posibles fuentes de provisión. Por ultimo, se muestran ejemplos de la ejecución de análisis paleoambientales y paleoclimáticos, desarrollados sobre la base de registros geológicos y biológicos de detalle, para arribar a una correcta reconstrucción de la formación de sitios arqueológicos y su resolución temporal.
\end{abstract}

\footnotetext{
ABSTRACT We present examples of geological research directed towards the resolution of archaeological problems. The existing approaches are characterized as either multi-disciplinary or inter-disciplinary, evaluating the degree of involvement with the specific archaeological questions being asked. We also remark the perceived limitations in this kind of approaches, which are basically related with differences in terms of temporal scales of analysis and with an absence of a scientific policy that encourages inter-disciplinary research. We emphasize the utility of physiographic, topographical, geomorphological, geological, geochronological, and pedo-sedimentary studies for an adequate contextualization of the archaeological record; likewise the requirement of a detailed knowledge of the nature of the inorganic raw materials used for the confection of the material culture, and of their possible provision sources. Finally, the conduction of paleoenvironment and paleoclimatic research, based on fine-grained geological and biological records, allows for a correct reconstruction of the formation processes of the archaeological record.
} 


\section{Intoduccíon}

La necesidad de trabajos de interrelación científica en el ámbito Arqueológico ha generado en los últimos años un creciente intercambio académico, en este trabajo con la disciplina geológica, que tiende a traspasar las fronteras de lo multidisciplinario para llegar al campo de la interdisciplina.

La importancia de la interdisciplina científica en Arqueología quedó de manifiesto en las Jornadas que, sobre Arqueología e Interdisciplina, organizó en 1993 el Programa de Estudios Prehistóricos en Buenos Aires y posteriormente durante la Mesa Redonda "El enfoque interdisciplinario en la resolución de problemas arqueológicos", organizada por el XII Congreso Argentino de Arqueología, en 1999.

Multidisciplina o Interdisciplina? Cuándo y Por qué? El aporte multidisciplinario es válido y útil cuando lo que se necesita, en la investigación arqueológica, es una mera descripción de variables asociadas al objeto de estudio, por ejemplo procedencia de materias primas, composición químico-mineralógica de un pigmento, petrología de un material lítico, composición mineralógica de pasta y antiplástico utilizada en la manufactura cerámica etc.

En tanto, en una investigación donde se procura el conocimiento de los procesos naturales y antrópicos y de su interrelación, por ejemplo, el uso del espacio $v s$. condiciones paleoclimáticas y paleoambientales o la referencia cronológica de eventos geológicos en relación a la formación de sitios y/o resolución arqueológica, aparece la necesidad de integrar las variables o datos recolectados por las diferentes áreas y el trabajo se transforma en interdisciplinario.

En el primer caso, el investigador maneja la información en forma individual, junta los informes "técnicos" de los especialistas, resume y plantea las conclusiones. En el segundo, varios investigadores trabajan conjuntamente para responder los objetivos planteados en el estudio desde sus distintas áreas de conocimiento.

Se presentan en esta oportunidad distintas contribuciones efectuadas desde la geología, discriminadas, sobre la base de su finalidad en multidisciplinaria (MD) e interdisciplinaria (ID), desde las de fuerte sesgo metodológico a las de tendencia valorativa, en la resolución de interrogantes en distintos contextos arqueológicos.

\section{Opinión de la Comunidad Arqueológica}

Con finalidad de averiguar si era o no necesario contar con una interrelación disciplinaria, conocer las áreas de mayor interés, destacar las ventajas y especialmente obtener respuesta que apuntaran a subsanar los actuales aspectos que dificultan el desarrollo de esta perspectiva, se realizó una encuesta durante la Mesa Redonda "El enfoque interdisciplinario en la resolución de problemas arqueológicos" del XII Congreso Argentino de Arqueología (Blasi y Zárate, 1999).

Las dificultades que se mencionaron como condicionantes de la investigación interdisciplinaria, además del problema presupuestario, fueron el lenguaje de comunicación, la definición de la escala de trabajo y el conocimiento básico.

Los resultados dieron cuenta que un $90 \%$ de los participantes habían realizado en alguna oportunidad un trabajo de interdisciplinaria (¿o multidisciplinaria?) y que la factibilidad de realización en los lugares de trabajo eran de un $70 \%$. Consultados sobre cuál, de entre un numero de disciplinas, presentaba la mayor dificultad de acceso, se destacó la Geología (Fig.1-A).

Por último y acerca de las temáticas que los arqueólogos priorizaban como de mayor necesidad para el desarrollo de sus investigaciones, el $25 \%$ de los encuestados manifestaron necesitar asesoramiento acerca de las condiciones paleoclimáticos y paleoambientales del contexto analizado. (Fig.1-B)

La dificultad en obtener aportes geológicos se debe en gran medida a vacíos en una especialidad disciplinaria como es el escaso número de geólogos del cuaternario y sedimentólogos de ambientes actuales, que son los que están más consustanciados con las problemáticas del intervalo Pleistoceno-Holoceno, intervalo temporal en el que se desarrollan las ocupaciones humanas del territorio Americano. Por otra parte, las políticas científicas estatales, no valorizaron, sino hasta hace muy poco, las contribuciones de sesgo multidisciplinario e interdisciplinario, hecho que desalentó este tipo de trabajos.

\section{Aportes a investigaciones arqueológicas multidisciplinares}

Se describen a continuación algunos aportes geológicos de diversos alcances, que fueron motivados por una gran variedad de planteos ar- 
A

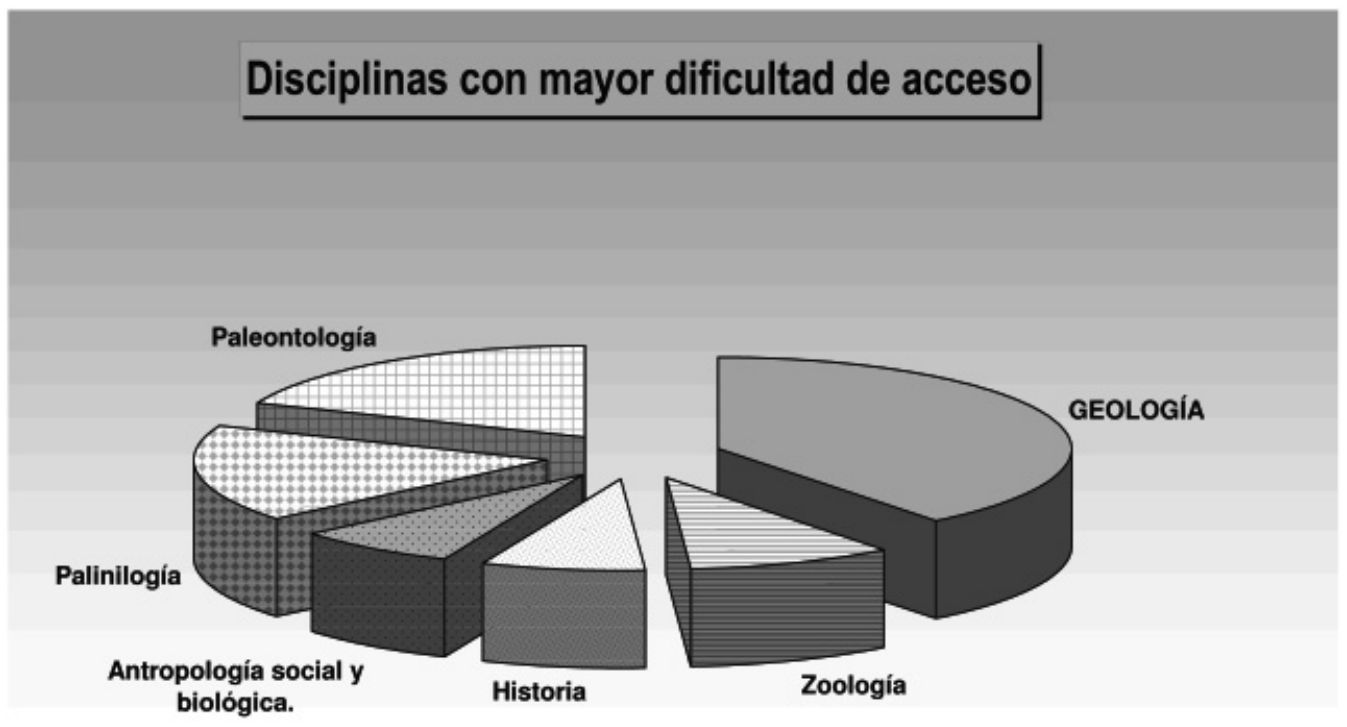

Figura 1.- Resultados de la encuesta en Mesa Redonda: "El enfoque interdisciplinario en la resolución de problemas arqueológicos" del XII Congreso Argentino de Arqueología. A-Disciplinas con mayor dificultad de acceso. B-Necesidad de asesoramiento en determinados campos del conocimiento
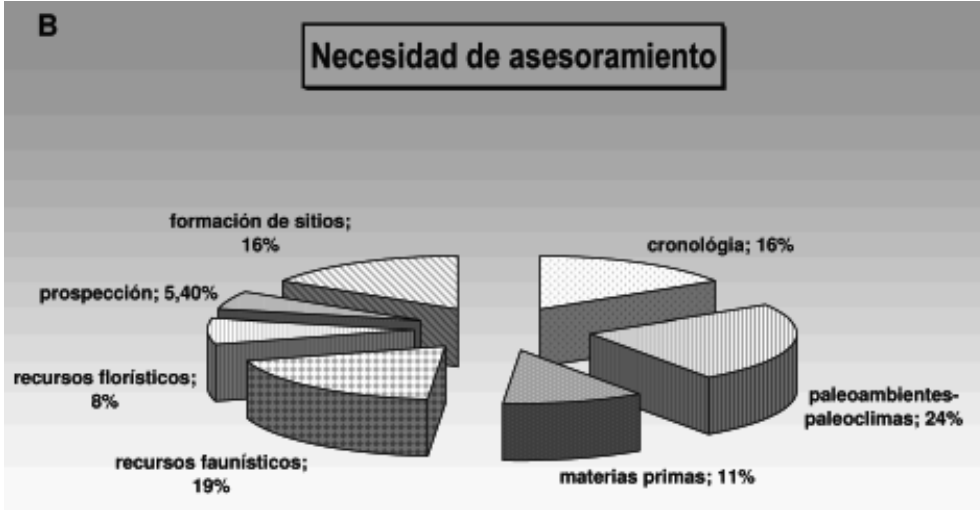

queológicos y realizados como informes técnicos y trabajos científicos con la aplicación de variada metodología y análisis.

\section{Materias Primas}

Las materias primas inorgánicas, utilizadas para la realización de la cultura material indígena, se estudian a través de análisis ceramológicos, análisis líticos y análisis de pictografías, en muchos de los casos con asesoramiento geológico.

En estudios ceramológicos son frecuentes las preguntas eson las materias primas utilizadas para la manufactura cerámica locales o no? ¿Es posible discriminar, a través de la composición de una pasta $y / 0$ antiplástico, piezas de elaboración local de foránea?

Para dar respuesta a este interrogante se deben enfocar tres aspectos: la naturaleza composicional de la pasta (P), de las sustancias agregadas o antiplás- tico (AT) y la naturaleza mineralógica y disponibilidad de material en las inmediaciones del sitio.

Las metodologías más empleadas son, el análisis mineralógico del AT del material cerámico mediante observaciones de corte delgado de la pasta (Fig. 2-G), por difracción de rayos X de muestra en polvo (Fig.2-E) y estudio de hojas geológicas del área (Fig.2-A).

En cuanto a la materia prima local, se llevan a cabo análisis de sedimentos y/o sedimentitas aflorantes a escala local, ya sea en paredes rocosas, barrancas y depósitos superficiales procedentes de arroyos, playas, medanos, etc. En este caso se utilizarán metodologías sedimentológicas como, análisis composicional de las fracciones granulométricas bajo estereomicroscopio (lupa) (Fig.2-B), petrografía a grano suelto (Fig. 2-C) y/o de cortes delgados por impregnación con resinas "epoxy", difracción de rayos $\mathrm{X}$ de muestras orientadas (na- 

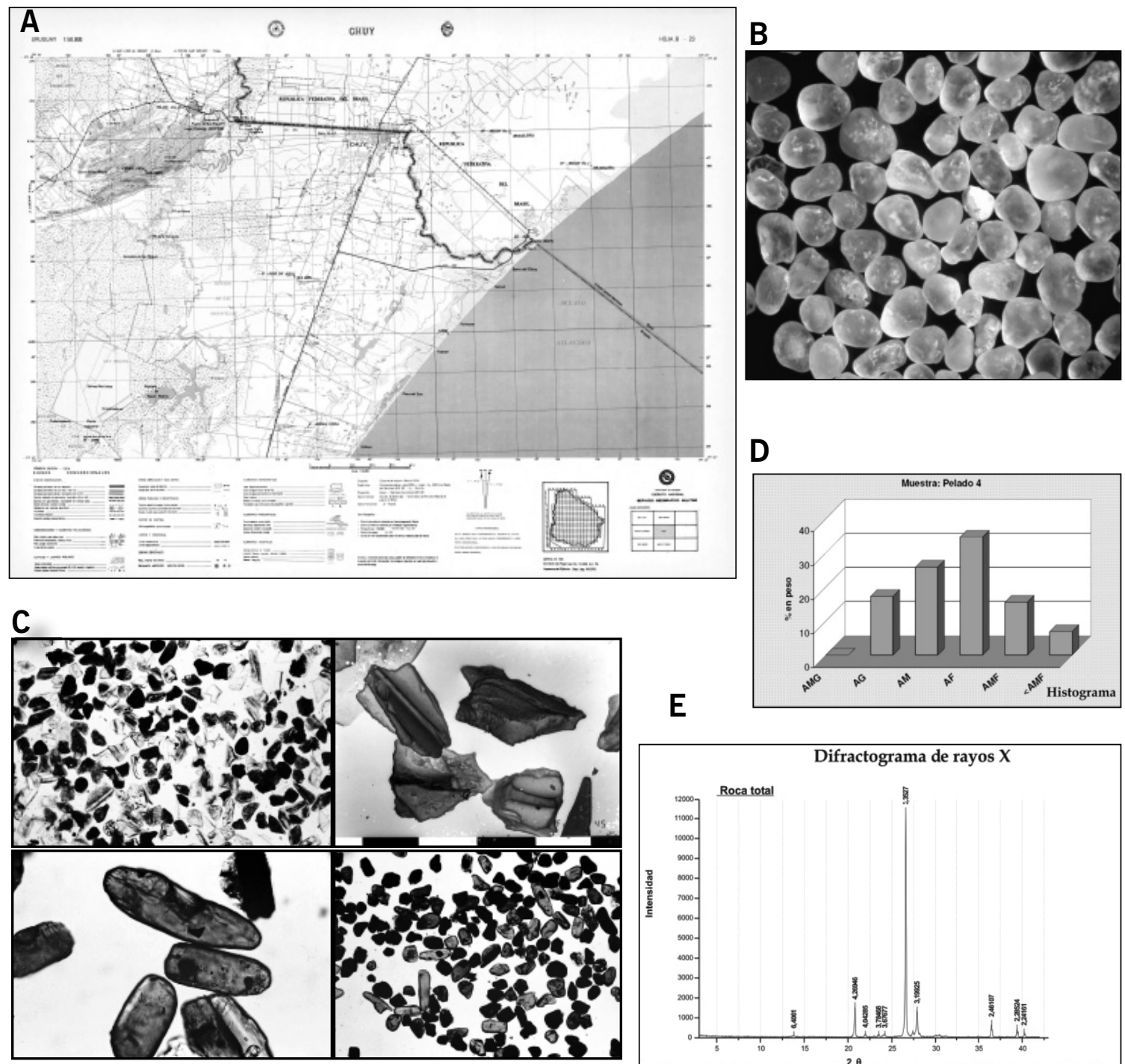

$E$

D

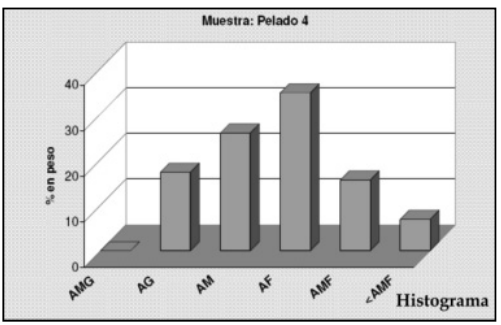

$\mathbf{F}$
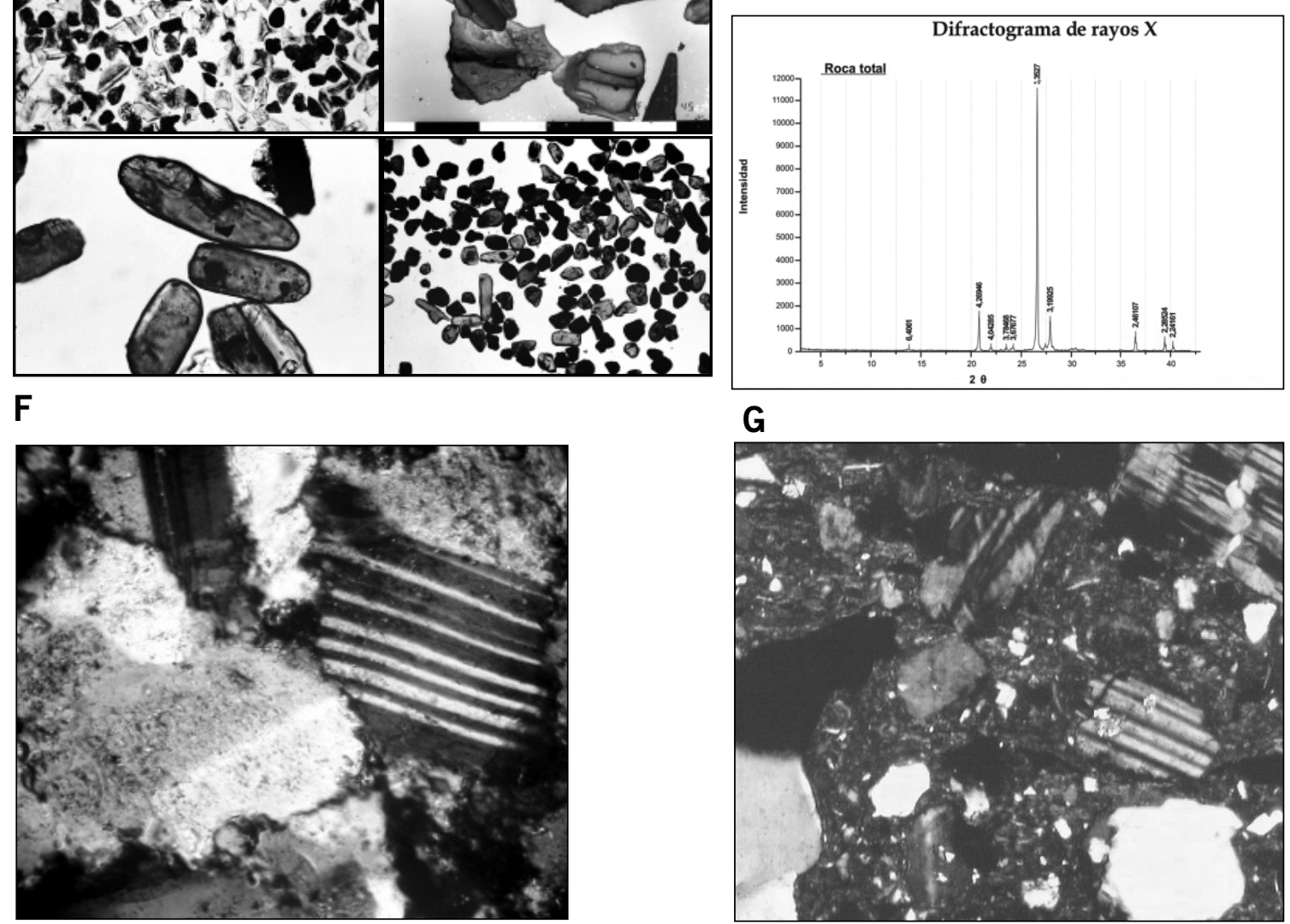

G

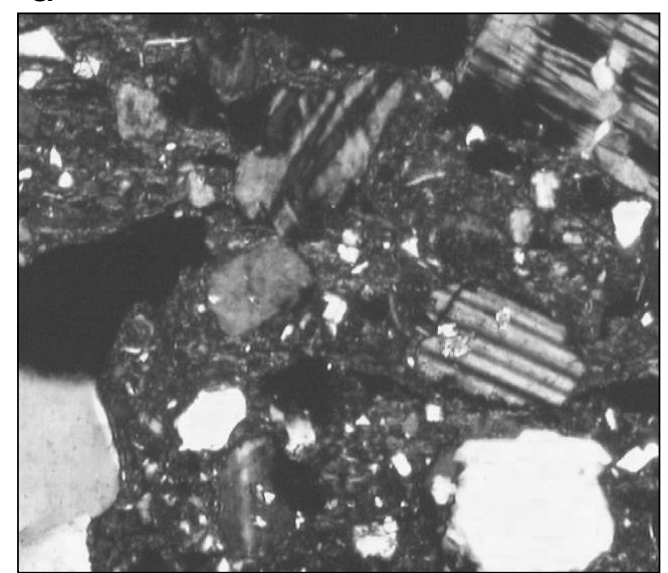

Figura 2.- A- Ejemplo de Hoja Topográfica escala 1:100.000. B-Análisis de fracciones granulométricas bajo Lupa. C- Análisis petrográfico a grano suelto por microscopia de polarización (minerales livianos imagen superior -minerales pesados imagen inferior). D- Histograma de un análisis granulométrico. E- Difractograma de rayos $X$ muestra en Polvo. F- Análisis Petrográfico de corte delgado de resto lítico. G- Corte delgado de cerámica 
turales, glicoladas y calcinadas) y los análisis granulométricos estándares, (Fig.2-F).

Limitaciones interpretativas a tener en cuenta: La composición de la pasta cerámica esta sujeta a los cambios producidos durante la cocción de la pieza, por lo que la ausencia de un tipo de argilomineral que pierde su estructura cristalina a temperaturas superiores a $500^{\circ} \mathrm{C}$ no es indicativa de su ausencia en los materiales explotados. Los antiplásticos intencionales han sido descriptos y estudiados frecuentemente por ser en muchos casos excelentes indicadores de procedencia. Sin embargo, la determinación de éstos, a excepción de los granos de tiesto molido y de mica de gran tamaño, se torna muy difícil, ya que el material con el que se realiza "la pasta" (un fango arenoso, un fango, etc.) puede contener naturalmente granos minerales que se destacan entre las partículas de tamaño fino, por lo que la diferenciación entre unos y otros requiere de planteos puntuales. En cada caso particular habrá que tener en cuenta distintos aspectos como naturaleza de los granos (clásticos mono o poliminerales, bioclásticos, etc.), tamaño, grado de redondeamiento o angulosidad, cantidad y composición mineralógica.

Por su parte, el resultado valorativo del análisis de las hojas geológicas se enfrenta con problemas de escala ya que la exposición de un material apto para la manufactura puede escapar a la escala del mapeo. Así, una veta, una capa de escasos centímetros de espesor o los materiales superficiales, que aparecen en la Carta Geológica bajo la denominación de depósitos cuaternarios /Cenozoicos /actuales, carecen, en éstos mapas utilizados de su descripción petrográfica. En este caso es importante realizar un muestreo y análisis de sedimentos de dunas, lagunas, ríos y arroyos o de sus barrancas en la región del sitio arqueológico. Se analizará la composición de los sedimentos y se podrá entonces vincularlos a los afloramientos de roca distinguidos en las Hojas Geológicas (Fig.2-A) observadas y cotejarlos con los cortes de cerámica. (Blasi 1985, 1987; Meroni y Blasi 1991, 1994).

En estudios de material lítico y ante la pregunta ¿de que material petrográfico se trata?¿Hay en el área? es posible realizar informes geológicos con la clasificación litológica del material por observación bajo lupa y en algunos casos a través de estudios petrográficos en cortes delgados (Fig.2-F). Pueden realizarse además, estudios de algunas propiedades físicas como dureza, maleabilidad, etc. y estudios de texturas superficiales en análisis de microscopía electrónica. En cuanto a si la fuente de provisión es local, regional o alóctona, es necesario hacer valoraciones del marco geológico de emplazamiento del sitio arqueológico mediante el análisis de Hojas Geológicas y muestreos o recolecciones superficiales de material en ambientes sedimentarios actuales. Limitaciones en la observación e interpretativas: en muchos casos la imposibilidad de hacer un corte delgado lleva a tener que reunir los materiales en tipos litológicos amplios de denominaciones generales como Plutonitas, Piroclastitas, Metamorfitas, Silicitas y Silicitas orgánicas. Respecto a la vinculación de las litologías líticas encontradas en los materiales con las rocas expuestas en el área, se debe tener en cuenta las limitaciones que se mencionaron anteriormente, en fuentes de provisión de material para la manufactura cerámica. Esto es a la posible existencia en la comarca de material no mapeable por su reducido tamaño de exposición.

En cuanto a investigaciones que han tenido por objeto determinar la composición mineralógica de pinturas utilizadas en cerámica $\boldsymbol{y}$ en arte rupestre se cita, sólo como ejemplo, el trabajo de Iñiguez y Gradín (1977) quienes realizaron el estudio de los pigmentos utilizados en la pictografía, mediante un análisis por difracción de rayos $\mathrm{X}$ muestra en polvo de los pigmentos raspados y molidos, de la Cueva de las Manos. Es estudio permitió reconocer los pigmentos utilizados, sin embargo no se pudieron vincular con los componentes minerales de materiales aflorantes en las barrancas del río Pinturas, uno de los objetivos del trabajo.

\section{Marcos Referenciales}

Son comunes los asesoramientos efectuados con la finalidad de contextualizar el sitio arqueológico en marcos geográficos, fisiográficos, topográficos, geomorfológicos, geológicos, geocronológicos y pedosedimentarios, entre otros.

Es importante resaltar que se debe realizar, previo al estudio del marco solicitado, una jerarquización de escalas de análisis o niveles de resolución espacial, ya que dependerá de la escala de observación el alcance o extensión que podrán brindarse con los mismos.

Escala regional y zonal: En esta escala se analiza el marco de referencia Geológico con identificación de las Unidades litoestratigráficas expuestas (Formaciones) que servirá, entre otros fines, para evaluar la disponibilidad de materias primas y la procedencia de los detritos en los materiales sueltos 
; del geomorfológico, pudiendo hacer inferencias en cuanto a la dinámica geomorfológica de la región en estudio y una cronología relativa de las unidades del paisaje, con el fin de brindar un marco geomorfológico de referencia para la localidad y un conocimiento de los procesos geomorfológicos intervinientes quizás en la erosión, removilización o sepultamiento de sitios (Blasi 1996, Zarate et al. 2000, Blasi y Zarate 2002).

Escala local: El análisis estratigráfico de los depósitos a escala local puede abarcar un área de unos $400 \mathrm{~m}^{2}$ que permita conocer la estratigrafía de los depósitos de cubierta, el cual servirá posteriormente para correlacionar con interpretaciones realizadas a escala de sitio (Zarate et al. 2000).

Escala de sitio: Los marcos referenciales a escala de sitio se constituyen con el levantamiento de perfiles estratigráficos de las excavaciones, cuadriculas y sondeos. Así, mediante la construcción de columnas sedimentológicas o pedo-sedimentológicas (Fig 6-C) y/o perfiles (Fig.9-D) sedimentológicos en área de pocos $\mathrm{m}^{2}$ que, individuales o integradas (Fig. 9-D) nos permite estimar varios aspectos, como variaciones depositacionales (alternancia de estabilidad (pedogénesis), depositación, erosión, tasas de agradación, cambios hidrológicos, etc.), paleoambientales, cronología relativa o absoluta y valoración del grado de resolución del registro arqueológico (Blasi 1985, 1987, Zarate et al. 1997, Zarate et al. 2000, Blasi y Zarate 2002).

\section{Conservación de Objetos Arqueológicos}

En el marco de un proyecto de preservación de las piezas egipcias (Fig.3-A) del Museo de La Plata, Argentina, la Fundación Getty concedió un subsidio para financiar un diagnóstico sobe el estado de conservación de la colección. El especialista en conservación se encontró con el siguiente interrogante ¿por que el material de las piezas arquitectónicas del Templo Aksha - Ramses II es tan deleznable y que lo ocasiona? ¿Que relación tiene la roca tallada con las "areniscas de Nubia"?

Figura 3. - A- Pieza del Templo Aksha en Museo de La Plata (Buenos Aires-Argentina). B-Corte delgado de la roca impregnada con resina con colorante. C-Microfotografía SEM: vista contacto entre el borde de grano de cuarzo y la matriz caolinítica. Nótese la desorientación de las placas. C- Microfotografía SEM: Detalle sector con caolinita autigénica, tamaño del paquete y de un individuo

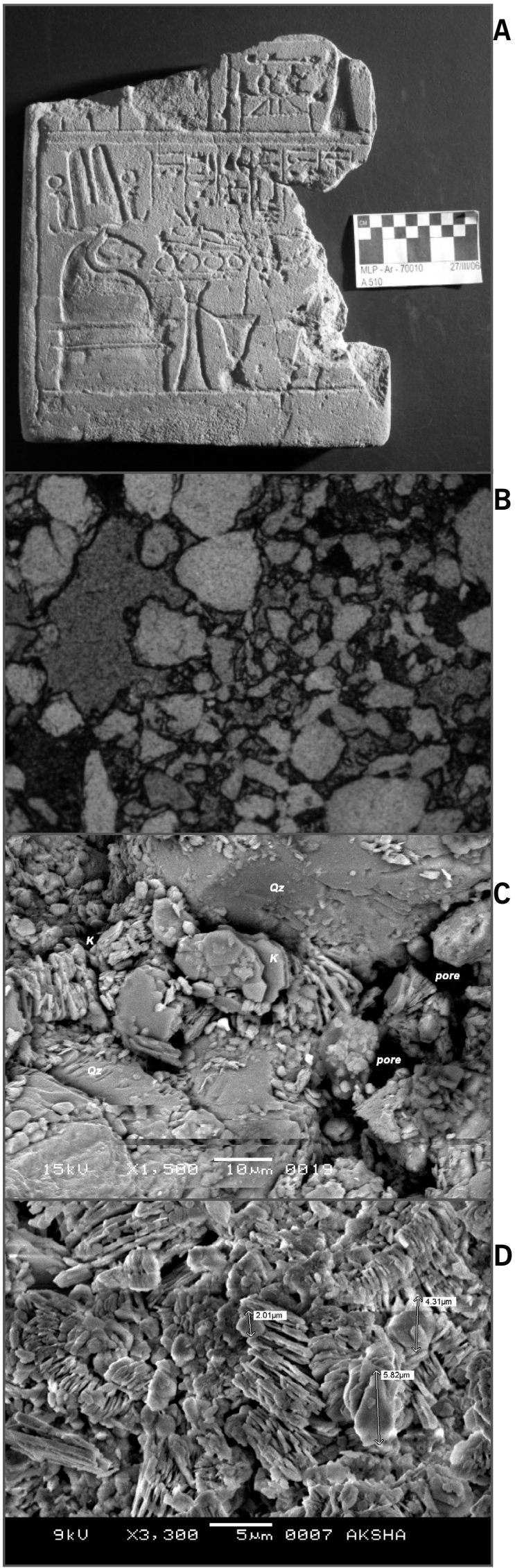




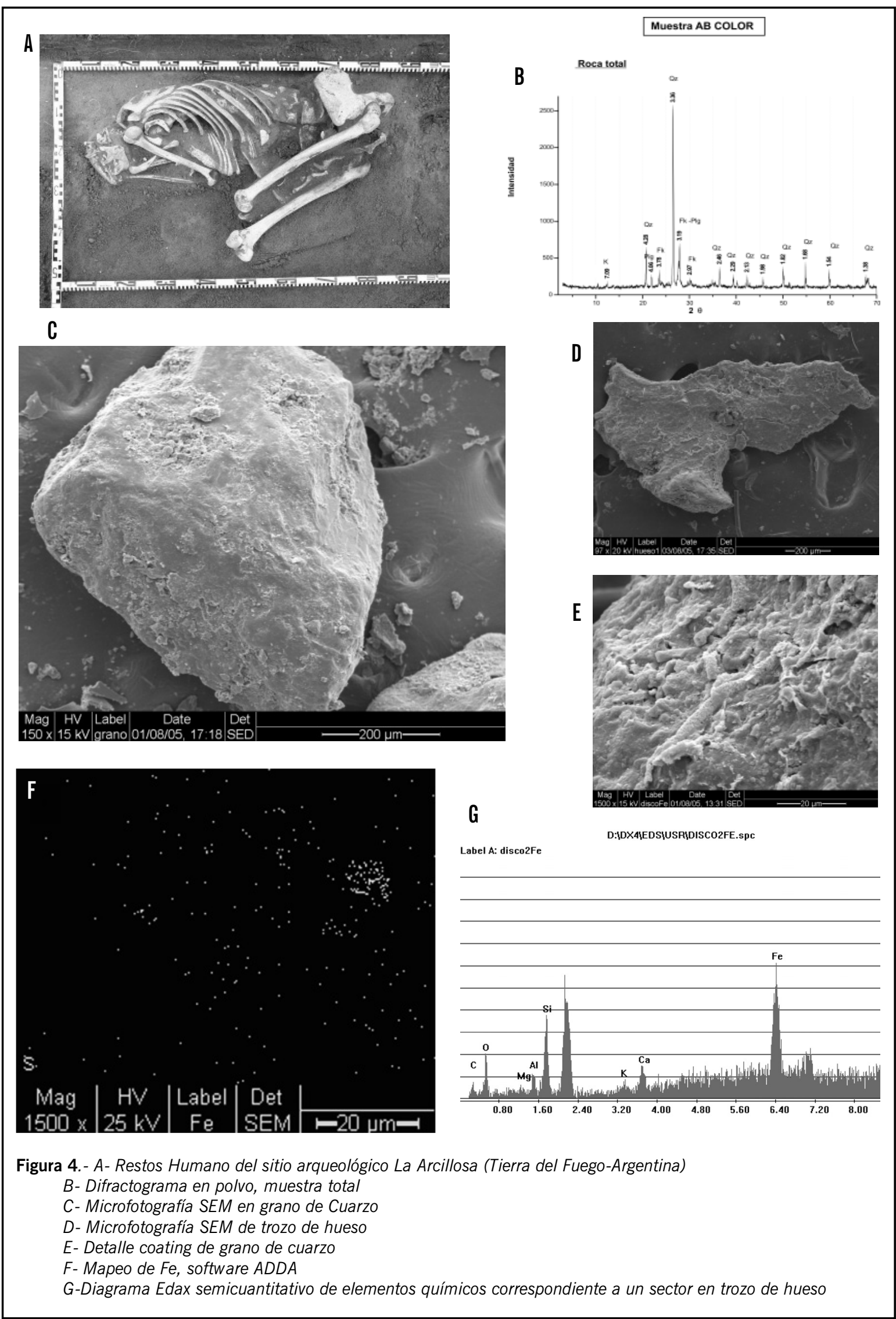


Para dar respuesta a estos interrogantes, se realizaron análisis por microscopía de polarización de cortes delgados (Fig.3-B), microscopía electrónica de barrido normal y microscopía electrónica de barrido método BSE (Fig.3-C y D), difractometría de rayos $\mathrm{X}$ de muestra en polvo y análisis Edax con utilización del software ADDA (Ametrano et al. 2006). Las propiedades litológicas encontradas en la roca analizada permitieron informar sobre la debilidad de la fábrica clástica y la susceptibilidad de desgranarse por perdida de la escasa y lábil cementación caolinítica (Ametrano et al. 2006).

\section{Restos Humanos}

Durante la excavación del sitio arqueológico La Arcillosa, (Saleme et al. 2005) se observó una diferencia de coloración en los materiales hospedantes de los restos óseos humanos (Fig.4-A), con preferencial concentración en inmediaciones de la pelvis y piernas. El planteo fue ¿a que se debe la coloración rojiza y que motiva este aumento de coloración en forma puntual en un mismo sedimento?

En virtud de esto se realizó el análisis granulométrico de muestras denominadas sin color y con color, correspondiente al sedimento más rosado (en el sector de la pelvis y piernas) y de tonalidad más clara (sector restante) respectivamente. Los resultados del análisis mecánico se representaron con histograma y clasificaron texturalmente los sedimentos.

La composición mineralógica se determinó mediante análisis petrográfico por estereoscopia binocular y microscopía de polarización de grano suelto.

Para la determinación de composición se utilizó difractometría de rayos X de muestra en polvo (Fig.4E). Se analizaron varios clastos (hueso y granos) de la muestra "con color", por microscopia electrónica Microscopio (SEM) Philips 505, detector EDAX con potencial a $15 \mathrm{Kv}$ (Fig.4- B, C y D). Se utilizó un software que permitió realizar el mapeo de elementos por barrido (mapa de elemento) y se obtuvieron imágenes con el software ADDA. (Fig.4-F).

Se pudo arribar a la conclusión que el sedimento eólico, de composición granítico-volcaniclástica que rodea el esqueleto, pertenece a un mismo depósito en todos sus puntos. Así como también, que la diferente coloración rojiza cercana a la zona de pelvis, rodillas y piernas, se debe a la existencia en la superficie de los granos, principalmente en irregularidades, de un pigmento de hierro (óxidos y/o oxido- hidróxidos de hierro: Goethita-Hematita?) de depositación irregular a veces muy puntual y de escasa dimensión del orden de escasos micrones. Y finalmente que el pigmento o recubrimiento que da la coloración rojizo-rosada provendría del cuerpo humano ya sea como vestigios de pintura (hematita) de vestimentas o de la piel. De ser así, quedaría aun por entender el significado de ésta localización preferencial de éstos.

\section{Aportes a investigaciones arqueológicas interdisciplinarias}

A continuación expondremos algunos ejemplos en donde la investigación arqueológica se desarrolló dentro de un encuadre más amplio en el cual el enfoque interdisciplinario fue la metodología imprescindible. Estos son un claro ejemplo de la interacción concreta y de una efectiva integración de los resultados.

A-Sedimentación y pedogénesis en un alero rocoso (Piedra Museo, Santa Cruz, Argentina) (Blasi y Zarate 2002).

En la localidad Piedra Museo, el alero rocoso ubicado en el NE del macizo del Deseado, Provincia de Santa Cruz, Argentina (Fig.5-A), fue ocupado por el hombre en repetidas oportunidades desde hace unos 11.000 años A.P. (Miotti et al.1999).

La aproximación geoarqueológica fue utilizada con los siguientes objetivos: brindar un marco estratigráfico de referencia para las ocupaciones humanas, inferir la evolución y génesis del contexto sedimentario, reconstruir condiciones paleoambientales a partir de los indicadores geológicos, evaluar la dinámica del paisaje, analizar la disponibilidad y calidad de materias primas líticas y evaluar los procesos de formación de sitios para estimar el grado de resolución del registro arqueológico (Zarate et al. 2000).

Para comprender la variación de texturas se ponderó la frecuencia relativa de los aportes extracuencales, intracuencales y posdepositacionales por reorganización pedogenética, (Fig.5-B y C). Se realizaron conclusiones particulares de los depósitos del alero.

El perfil de suelo se desarrolló a expensas de un material parental de aporte mixto (intracuencal -extracuencal) de granulometría casi constante y con variaciones en la importancia relativa de los aportes durante el proceso acrecional. 
Entre las consideraciones generales se definió el inicio de la sedimentación ca. 13.000 años ${ }^{14} \mathrm{C}$ AP, con baja tasa de aportes eólicos intracuencales hasta los 10.000 AP (método ${ }^{14} \mathrm{C}$ ). En este lapso de tiempo la superficie se habría modificado por acreción en presencia de un suelo incipiente que sirvió de sustrato a los grupos humanos que ocuparon el alero en ese momento. Posteriormente, entre 10.000 y 7.000 años AP se habría producido estabilidad geomorfológica, disminución de la tasa de sedimentación e incremento de la pedogénesis. El aporte sedimentario habría sido de naturaleza intracuencal por derrumbe de la roca del alero en las arenas, con escaso aporte eólico local y eólico extracuencal. Entre 10.000 y 7.000 años AP habría predominado la pedogénesis sobre la sedimentación. A partir de 7.000 AP (método ${ }^{14} \mathrm{C}$ ) se inició la diferenciación de los diversos horizontes del perfil, proceso que continuó durante el Holoceno. La carpeta arenosa superior, sin rasgos pedológicos, se considera de una génesis relativamente reciente y se la vinculó a los procesos de aridización de los últimos 100 años.

\section{B-Sedimentación Holocena en los Baña- dos de Santa Teresa y el Registro Arqueoló- gico, Planicie Costera de Departamento de Rocha, Uruguay (Blasi et al. 2005).}

Este estudio forma parte de investigaciones tendientes a la reconstrucción paleoambiental-paleoclimática de la planicie costera del NE uruguayo durante el Holoceno, en vinculación con el registro arqueológico del área.

La geología de la cuenca de la Laguna Negra fue estudiada a partir de registros de alta resolución (proxy record), mediante análisis geoquímico, biológico de restos orgánicos silíceos y carbonáticos (Fig.6-B y D), sedimentológico y de datación radiocarbónica (Fig.6-E).

La manifestación arqueológica prehistórica más sobresaliente para esta región, es la presencia de construcciones antrópicas en tierra denominadas "cerritos de indios" (Fig.6-A).

A través de los resultados obtenidos del estudio de los depósitos superficiales y subsuperficiales del Bañado Santa Teresa (alrededores de la Laguna Negra), Departamento de Rocha - Uruguay (Fig.6$\mathrm{C})$, se reconocieron 3 unidades depositacionales de edad Holoceno Medio - Holoceno Tardío cuyas características permitieron considerar la existencia de una serie de subambientes ácueos de depositación de baja energía para el lapso.

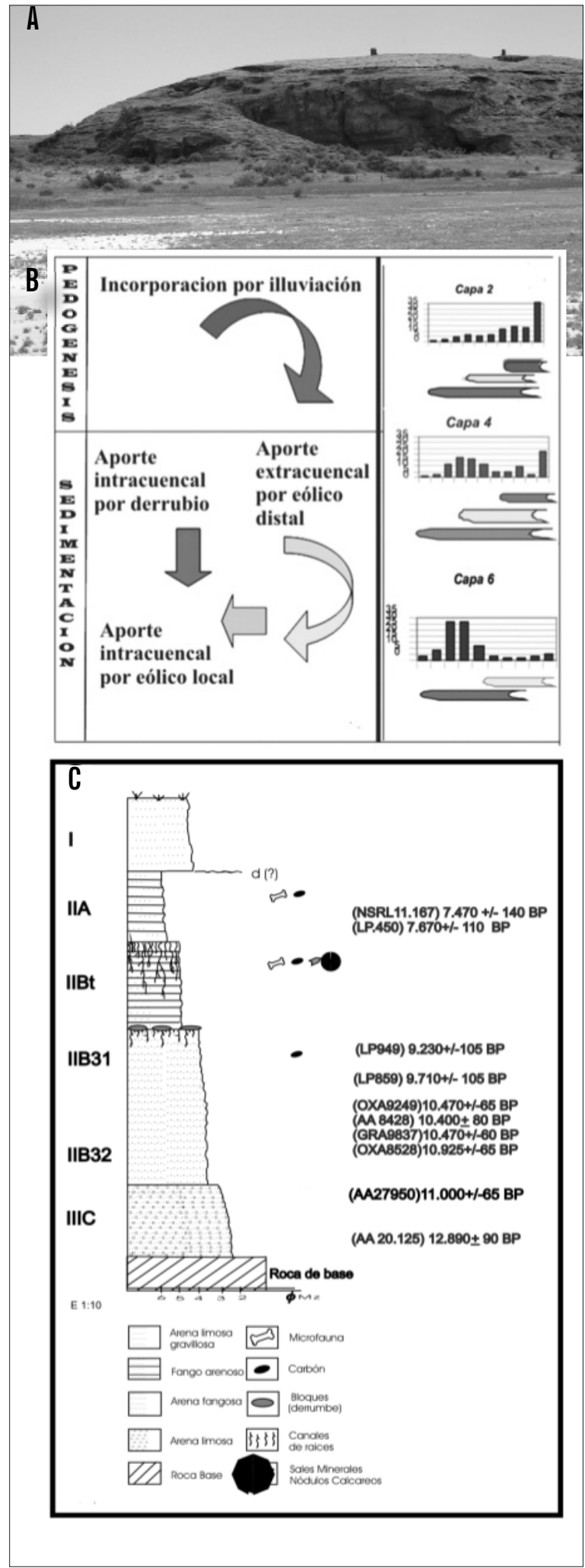

Figura 5.- A- Imagen Sitio Arqueológico Piedra Museo (Santa Cruz-Argentina). B-Discriminación de aportes sedimentarios y pos-depositacionales, (derecha). Influencia de los diferentes aportes en la distribución granulométrica de las diferentes capas, (izquierda).C-Columna Pedo-Sedimentaria 
A

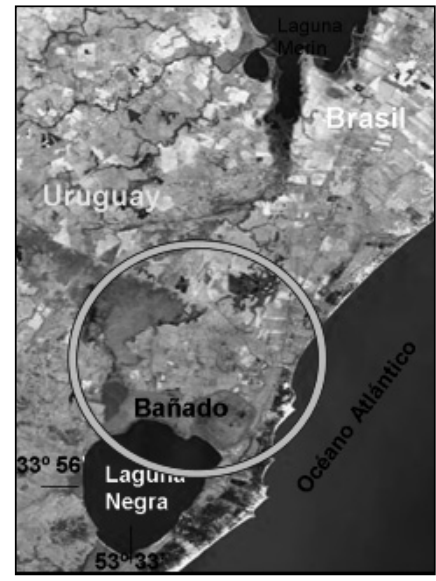

B
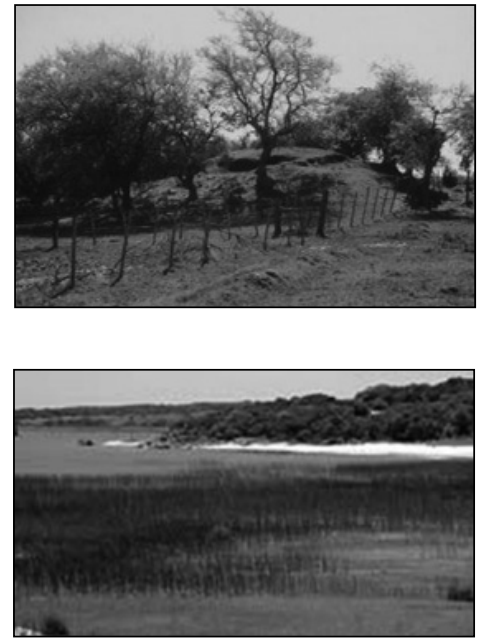

$\mathrm{E}$

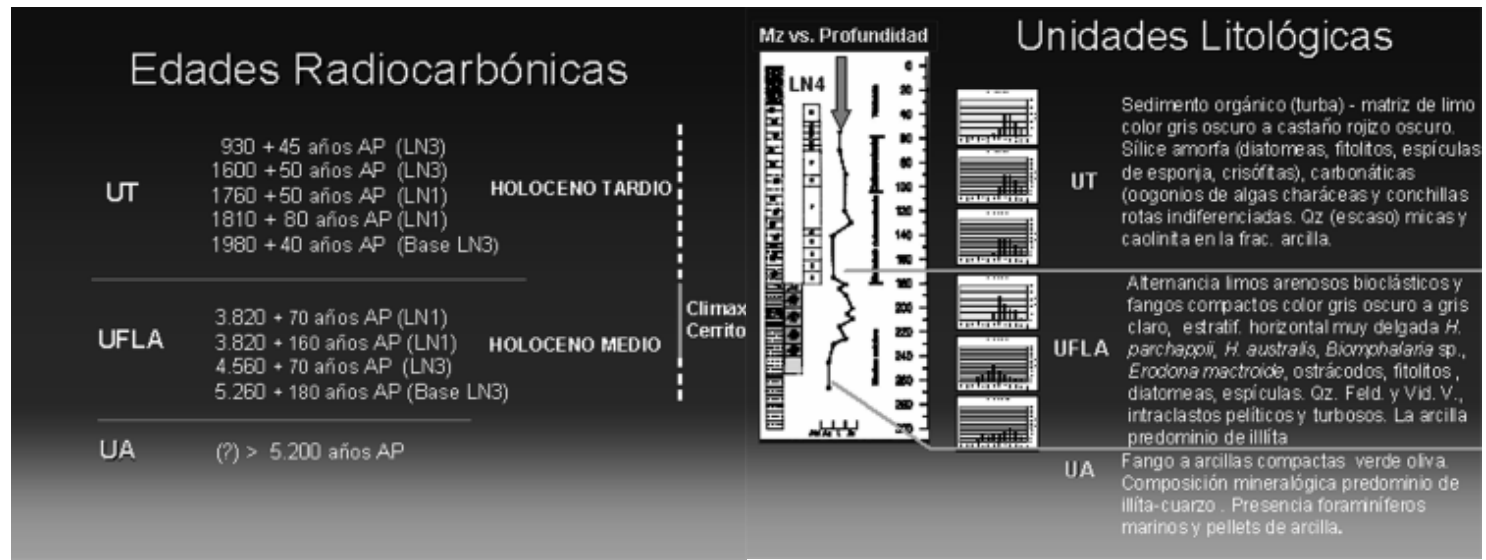

Figura 6.- A- Ubicación de Laguna Negra y Bañado de Santa Teresa. B- Imagen deCerrito de Indios y Laguna Negra (Rocha-Uruguay). C-Análisis de partículas biosilíceas. D-Análisis de Contenido de células C3/C4. E-Resultados análisis Sedimentológico y Cronológico de las unidades litológicas reconocidas

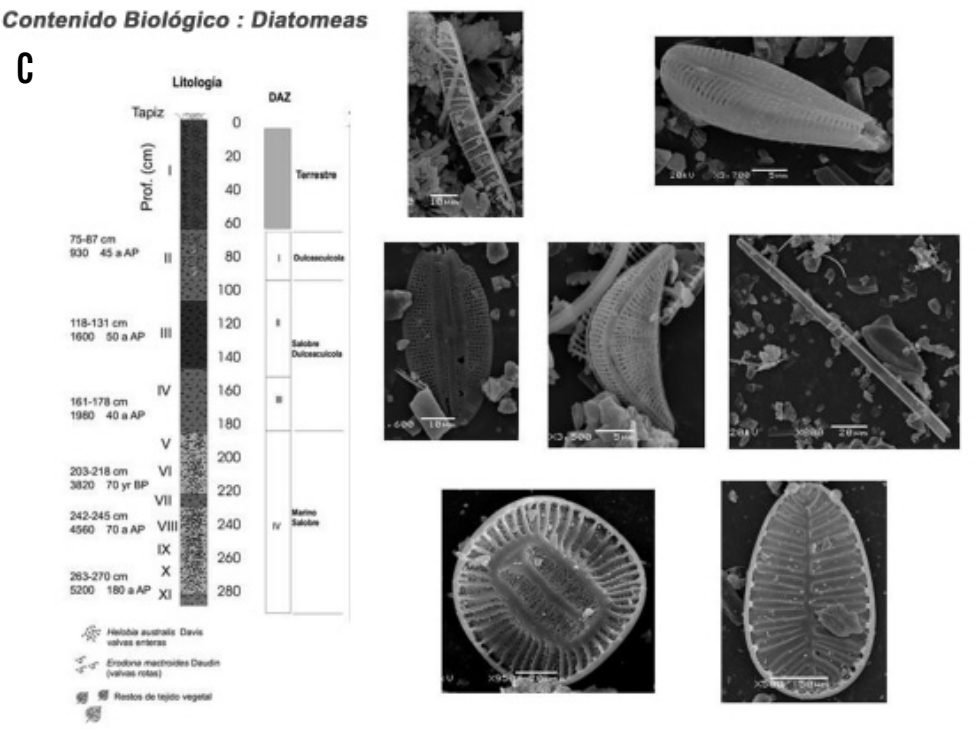

\section{Degistro silicofitolitos: clima}

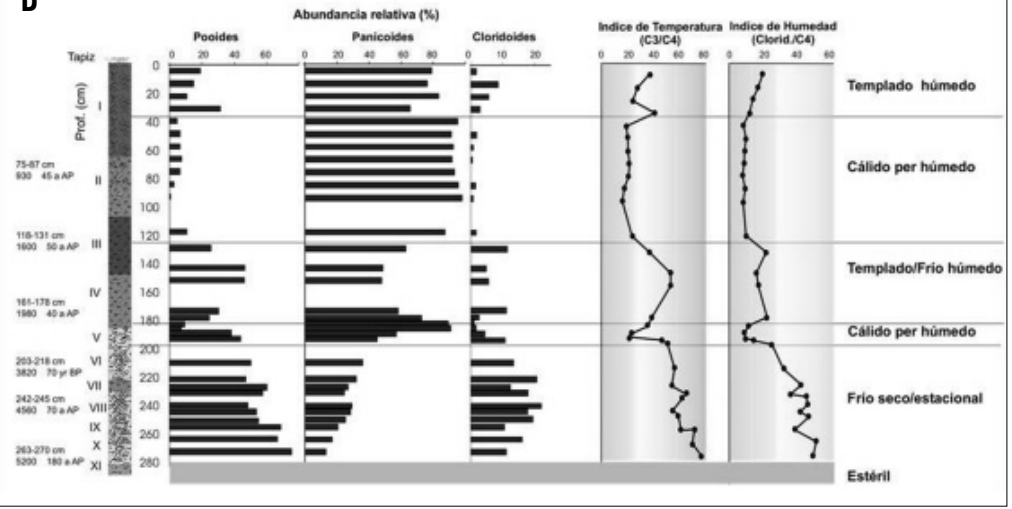




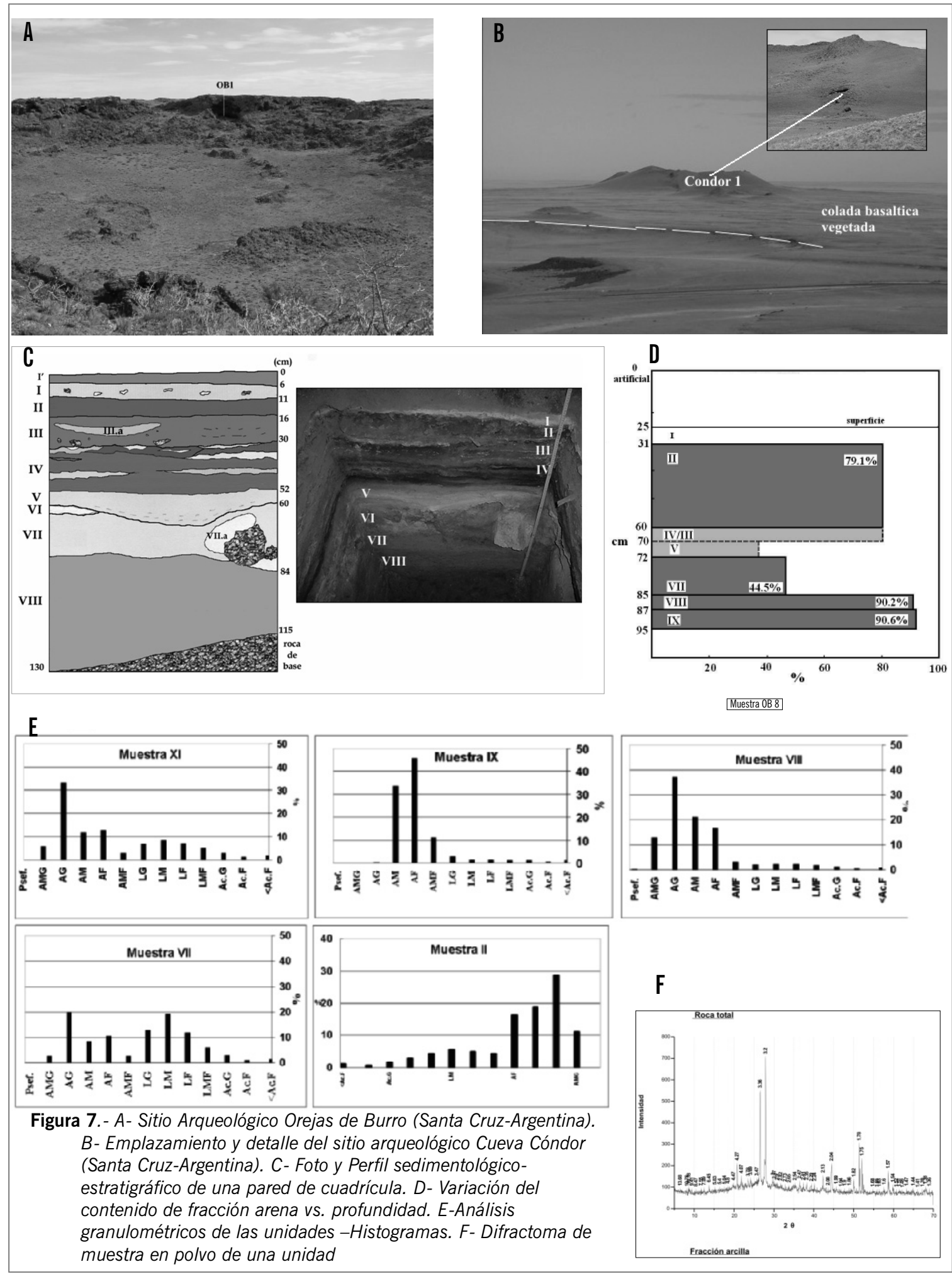

Se propuso como hipótesis de trabajo, para continuar con estas investigaciones, la existencia de un humedal "continuum " desde el máximo transgresivo y la ocurrencia de procesos mareales en la formación de subambientes eurihalinos-mixohalinos durante el Holoceno medio (marismas-pantanos salobres) y su evolución a bañados turbosos dulceacuícolas hacia el Holoceno tardío.

En este supuesto, los grupos humanos prehistóricos que ocuparon, colonizaron y construyeron 
“cerritos" en la cuenca de la Laguna Negra, debieron desarrollar estrategias de explotación y ocupación vinculadas a la presencia de amplias extensiones de humedal (Blasi et al. 2005).

\section{C- Geoarqueología en Pali Aike: cueva} Orejas De Burro 1 (Barberena et al. 2005, 2006a).

El campo volcánico de Pali Aike (CVPA) es una de las regiones en las cuales se inicia la arqueología patagónica. Los trabajos desarrollados por Junius B. Bird a partir de la década de 1930 en las cuevas Fell y Pali Aike, entre otras, proveyeron el esquema empleado para comprender la historia cultural de Patagonia meridional.

Recientemente se comenzó a reevaluar la arqueología de esta región en función de preguntas de carácter biogeográfico, que enfatizan el análisis de la organización espacial de las poblaciones humanas. El proyecto general, en el cual se enmarca este trabajo, busca evaluar distintos aspectos de la organización geográfica de las poblaciones humanas que habitaron este espacio durante el Holoceno.

A nivel geoarqueológico específico, los objetivos se agruparon en tres aspectos. En primer lugar, el estudio de los procesos de formación del registro, a fines de estimar el agente de sedimentación responsable del aporte. En segundo, el establecimiento de la cronología relativa y/o absoluta de los depósitos, mediante análisis de superposición de estratos, discontinuidades naturales y antrópicas y dataciones numéricas, la que permite además, la valoración de la tasa de sedimentación en los diversos eventos e intensidad de las ocupaciones humanas. Por último, reconocer y evaluar varia-

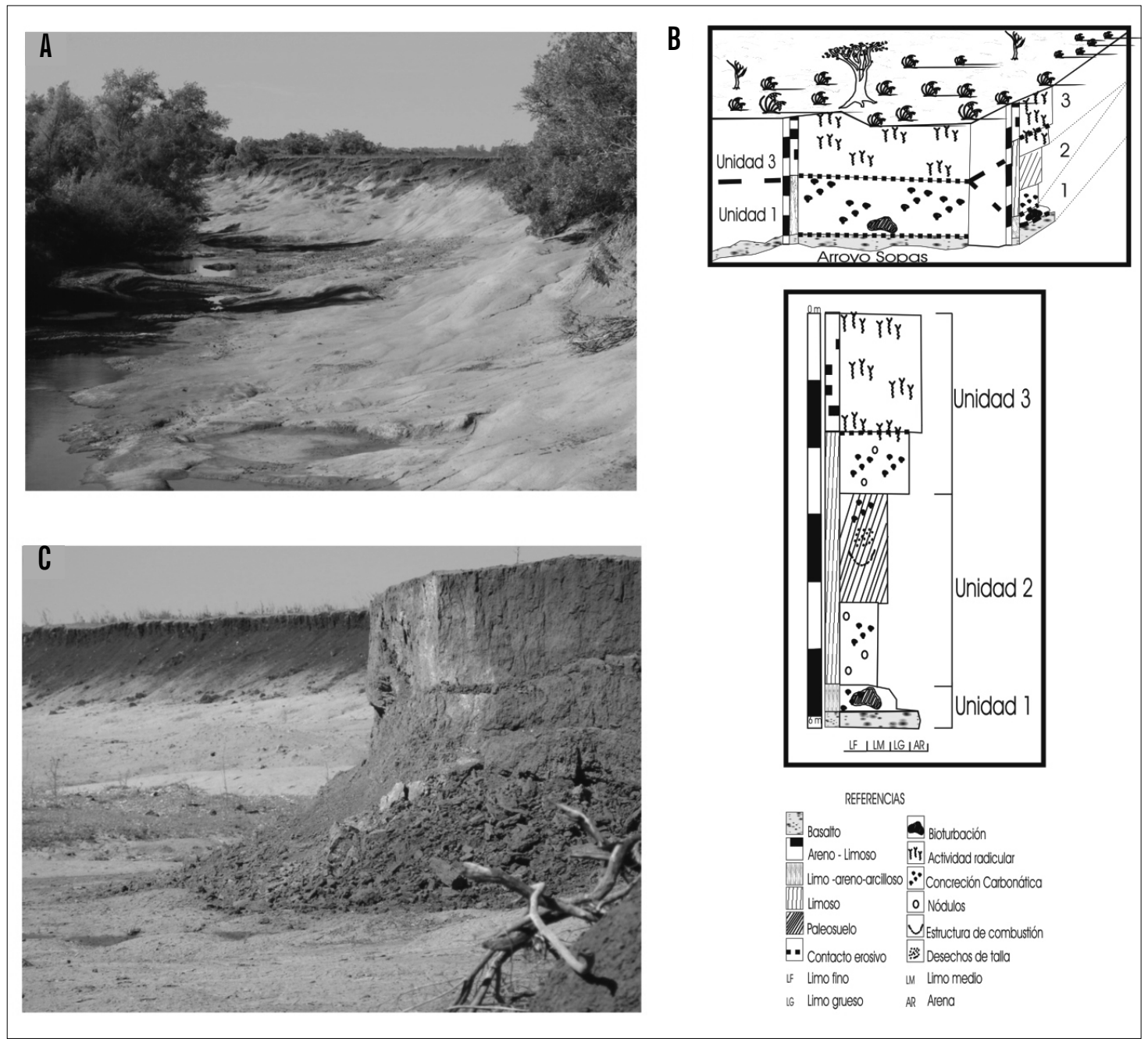

Figura 8.- A- Arroyo Sopas (Salto-Uruguay) Barranca Pleistocena. B-Correlación de columnas sedimentológicas en perfil integrado (arriba), columna sedimentológica (abajo). C- Vista de terraza Holocena 
ciones climáticas a escala local o de sitio a través de las variaciones sedimentológicas, naturaleza de los aportes y contenido biológico y su relación con fluctuaciones climáticas a escala zonal y regional.

Así y mediante el estudio de la naturaleza del material sedimentario hospedante de los registros biológicos y culturales de las Cuevas de Pal Aike y los procesos implicados en la génesis de los mismos, se llevo a cabo el trabajo geoarqueólogico sobre el sitio Orejas de Burro 1 (Fig.7-A). Se presenta en esta contribución algunas imágenes que muestran la información estratigráfica y geoarqueológica obtenida (Fig.7- D-E-F). En esta misma línea de trabajo se estudiaron las evidencias recuperadas en la Cueva Cóndor (Barbarena et al. 2006b), otro integrante del paisaje de cuevas del CVPA (Fig.7-B).

Las cuevas, constituyen excelentes "laboratorios naturales"pues poseen condiciones optimas para la formación y preservación de secuencias estratificadas, naturales y antrópicas, importantes tanto desde una perspectiva biogeográfica como geoarqueológica.

D- Aportes para una actualización de la correlación estratigráfica: Fm Sopas (Norte De Uruguay) - Fm. Lujan (Prov. De Buenos Aires) (Castiñeira et al. 2005).

En este trabajo, se dan a conocer los primeros resultados relacionados con la estratigrafía de los depósitos aflorantes en la localidad tipo de la Formación Sopas, con la finalidad de analizar los contextos de asociación del registro arqueológico y paleontológico durante la transición Pleistoceno-Holoceno en el norte de Uruguay.

La discontinuidad lateral de los depósitos aflorantes en las barrancas del Arroyo Sopas tramo inferior, nos llevo a la necesidad de agrupar las exposiciones en asociaciones, de tal forma de comprender el ordenamiento crono-estratigráfico de los distintos episodios de sedimentación en el área (Fig. 8-A a C). Se reconocieron al menos tres ciclos de sedimentación.

Al primer ciclo de sedimentación (A) definido en este trabajo se le asigna cronología Pleistocénica, siendo fértil paleontológicamente y estéril arqueológicamente. El segundo ciclo de sedimentación posee edades holocénicas (Holoceno Temprano - Holoceno Medio) Arqueológicamente los depósitos asignados a este segundo ciclo de sedimentación son los que pueden potencialmente integrar evidencias correspondientes a las etapas de exploración inicial, poblamiento y colonización prehistórica para el área. En tanto el tercer ciclo de sedimentación definido en este trabajo, es posiblemente asignable a la cronología de referencia sugerida pre - post conquista europea.

\section{Consideraciones finales}

No obstante las ventajas que surgen de la interrelación disciplinaria, se requiere aún de espacios de discusión, en pos de subsanar los aspectos actuales que entorpecen el acceso al asesoramiento en diferentes temáticas y la integración de grupos de trabajo.

En este sentido, se desea destacar que la colaboración interdisciplinaria promueve la formación e integración de equipos, la discusión valorativa desde los diferentes puntos de vista y el compromiso en la resolución del objetivo e interés común al que amplían y enriquecen.

\section{Agardecimientos}

La autora desea agradecer a los revisores por las sugerencias acercadas para mejorar el texto y al Prof. Dr. Celso Dal Ré Carneiro, editor de Terrae Didatica, por sus amables recomendaciones editoriales para optimizar esta presentación.

\section{Referencias bibliográficas}

Ametrano S., A. Blasi y C. Di Lello C. 2006. Petrologhy study of rock sample from Aksha Temple. (Inédito-no publicado).

Barberena R., A. Blasi y C. Castiñeira. 2006a. Geoarqueología en Pali Aike: cueva Orejas De Burro 1, Patagonia, Argentina. Magallania 34:103122. Chile.

Barberena R., A. Blasi y C. Castiñeira. 2006b. Geoarqueología y Biogeografía: El Registro De Cuevas en Pali Aike. En: Simposio Arqueología y Biogeografía. Diferentes escalas de análisis y líneas de evidencia. VI Jornadas de Arqueología de la Patagonia. Punta Arenas, 24, Octubre, 2005. Centro de Estudios del Hombre Austral, Instituto de la Patagonia, Universidad de Magallanes. Punta Arenas, Chile Bol. Resúmenes I, 28-29.

Barberena R., A. Blasi y C. Castiñeira. 2007. Geoarqueología y Biogeografía: El Registro De Cuevas En Pali Aike. Libro “Arqueología de FuegoPatagonia. Levantando piedras, desenterrando huesos... $y$ develando arcanos." Ediciones CEQUA. Punta Arenas, Chile. (En prensa).

Blasi A. 1985. Estudio Sedimentológico de un Perfil Arqueológico de la Prov. de Entre Ríos. Instituto 
de Inv. Arqueol. Dr. O. Menghin. Revista Sapiens 5: 31-56, Chivilcoy, Buenos Aires.

Blasi A. 1987. Análisis textural y composicional de los sedimentos superficiales de las inmediaciones de los sitios arqueológicos Palo Blanco, Asampay y Cínica. Prov. de Catamarca, (Informe técnico interno).

Blasi A. 1988. Estudio textural de la sucesión sedimentaria en la localidad arqueológico Cabo Blanco. Prov. de Santa Cruz. (Informe técnico interno).

Blasi A. 1996. Marco Geológico Regional y Geomorfológico local del Sector de la Sierra de Fiambalá, en relación a los emplazamientos de cementerios de La Ciénaga. (Informe técnico interno)

Blasi A., Rabassa J., Miotti L. y G. Cattáneo. 1997. Investigaciones geoarqueológicas en la Localidad Piedra Museo. En: XII Congreso Nacional de Arqueología Argentina, La Plata, 22, Septiembre, 1997. Universidad Nacional de La Plata. Facultad Ciencias Naturales y Museo, La Plata, Argentina., Actas T.1, p 9.

Blasi A. y M. Zárate 1999. El enfoque interdisciplinario en la resolución de problemas arqueológicos Mesa Redonda. En: XII Congreso Nacional Arqueología Argentina. La Plata, 22, Septiembre ,1997. Universidad Nacional de La Plata. Facultad Ciencias Naturales y Museo, La Plata, Argentina. Actas T II, p.375-383.

Blasi A. y M. Zarate. 2002. Sedimentación y pedogénesis en un alero rocoso (Piedra Museo, Santa Cruz). En: IX Reunión Argentina de Sedimentología. Córdoba, 21, Mayo, 2002. Asociación Argentina de Sedimentología (AAS). Argentina. Bol. Resúmenes, v I, p 81.

Blasi A. 2005. Diferencia de coloración de los sedimentos en El Sitio La Arcillosa: Un Indicador tafonómico?" Anexo 1. En: Salemme, M., G. Bujalesky y F. Santiago. La Arcillosa 2: la ocupación humana durante el Holoceno medio en el río Chico, Tierra del Fuego, Argentina. En: VI Jornadas de Arqueología de la Patagonia Punta Arenas, 24, Octubre, 2005. Centro de Estudios del Hombre Austral, Instituto de la Patagonia, Universidad de Magallanes. Punta Arenas, Chile Bol. Resúmenes I, p. 82.

Blasi, A. Diferencia de Coloración de los Sedimentos en el Sitio La Arcillosa: Un Indicador Tafonómico? 2007. En: Salemme, M., G. Bujalesky y F. Santiago. La Arcillosa 2: La Ocupación Humana Durante El Holoceno Medio En El Río Chico, Tierra Del Fuego, Argentina. Libro "Arqueología de Fuego-Patagonia. Levantando piedras, desenterrando huesos... y develando arcanos." Ediciones CEQUA. Punta Arenas, Chile. (En prensa).

Blasi A., C. Castiñeira, L. del Puerto, H. Inda,
R. Bracco y F.García-Rodríguez. 2005. Sedimentación Holocena en los Bañados de Santa Teresa y el Registro Arqueológico, Planicie Costera de Departamento de Rocha, Uruguay". En: XVI Congreso Geológico Argentino. La Plata, 20, Septiembre, 2005. Asociación Geologica Argentina. La Plata. Buenos Aires.Bol Resumenes, T IV, p. 289-297.

Castiñeira C., J. Fernicola, M. Zárate, A. Blasi, R. Bracco, H.Inda y L.del Puerto. 2005. Aportes para una actualización de la correlación estratigráfica: Formación Sopas (Norte Uruguay)-Formación Lujan (Prov. De Buenos Aires). Implicancias Arqueológicas. En: Primer encuentro de Discusión Arqueológica del noroeste Argentino "Arqueología de cazadores recolectores en la cuenca del Plata". Santa Fe, 11 de agosto, 2005. Museo Etnográfico y Colonial "Juan de Garay" Santa Fe. Argentina. Actas, v I, p 22-24.

Iñiguez M. y C.Gradin. 1977. Análisis Mineralógico por difracción de rayos X de muestras de Pinturas de la Cueva de las Manos, Estancia Alto río Pinturas., Santa Cruz. Relaciones, $v$ XI, Nueva. Serie: 121-129. Buenos Aires.

Miotti L., M.Vazquez y D. Hermo.1996. Piedra Museo, un Yamnagoo pleistocénico de los colonizadores de la meseta de Santa Cruz. El estudio de la arqueofauna: En: Simposio de Poblamiento. III Jornadas de Arqueologia de la Patagonia. Bariloche, 27 de mayo, 1996. Argentina. Bol. Resumenes, v I, p 113-136.

Pérez Meroni M. y A. Blasi. 1994. Sitio Arqueológico "El Ancla", Punta Indio, Prov. de Buenos Aires. Ensayo y Experimentación de Sedimentos Peliticos para la manufactura cerámica. En: XI Congreso de Arqueología Argentina. San Rafael, Mendoza, 23 de Mayo, 1994. Revista del Museo de Historia Natural, San Rafael, Mendoza. Argentina 14(1/4): p238-239.

Zarate M., A. Blasi y J. Rabassa. 2000. Geoarqueología de Piedra Museo. En: Simposio Modelos Paleoambientales durante la Transición Pleistoceno / Holoceno. La Plata, 4 Diciembre, 2000. Taller Internacional del INQUA La Plata y Provincia de Santa Cruz, Argentina. Guía de Campo, Cap.4 Ítem 4.2: 1-10.

Zaráte M, García, A. y A. Blasi. 1997. Condiciones ambientales entre los 14000 y los 5000 años ${ }^{14} \mathrm{C}$ AP en la Precordillera de Mendoza, Argentina. Localidad Agua de La Cueva. VI En: Congresso da Associação Brasileira de Estudos do Quaternário (ABEQUA) e Reunião sobre o Quaternário da América do Sul, Curitiba, Paraná, Brasil, 27 de junio a 3 de agosto de 1997. Bol. Resúmenes v I, p 517-521. 